\title{
INFLUENCE OF NON-UNIFORMITY OF HEATING ON UPSETTING FORCE VALUE AND FORGING TIME IN FLASH-BUTT WELDING OF FLAT RING
}

\author{
A.V. MOLTASOV, S.M. SAMOTRYASOV, V.V. KNYSH, P.N. CHVERTKO and K.V. GUSHCHIN \\ E.O. Paton Electric Welding Institute, NASU \\ 11 Bozhenko Str., 03680, Kiev, Ukraine. E-mail: office@paton.kiev.ua
}

\begin{abstract}
One of the reasons of occurrence of stresses and displacements in the rings, manufactured by flash-butt welding $(\mathrm{FBW})$, is the non-uniform temperature field, which is formed as a result of electric current shunting. In the frames of the present work the temperatures in different characteristic spots at the final stage of fulfillment of FBW of steel ring in machine K724 were measured. Measurements showed that at the investigated stage of welding process the temperatures of controlled spots are little changed with time, i.e. quasi-stationary heat flow takes place. Function of temperatures was preset in the form of Fourier series around the circumferential coordinate, moreover as a result of symmetry the odd members of this series were not taken into account. It appeared sufficient to be restricted by four first terms of the series. Change of temperatures in radial direction, determined from the conditions of stationarity of heat flow, showed that the action of temperature field in the ring hinders approaching of edges being welded. It results in the fact that a part of upsetting force is consumed for overcoming the elasticity forces caused by non-uniformity of heating. For the steel 20 ring considered the upsetting force, required for formation of joint, increased by $16.5 \%$. Besides, the presence of temperature stresses increases the force striving to rupture a butt after welding, thus leading to the necessity to prolong the butt forging time by 4 s. 7 Ref., 4 Figures.
\end{abstract}

Key $\boldsymbol{y} \boldsymbol{o} \boldsymbol{r} \boldsymbol{d} \boldsymbol{s}:$ flash-butt welding, flat rings, temperature field, stationary heat flow, temperature stresses, upsetting force, forging time

One of the most efficient methods of joining the elements of structures of ring shape is flash butt welding (FBW). Machines for FBW [1] provide different value of upsetting force for welding of products. During development of technological modes of welding it is necessary to determine the value of edge approaching force to provide the sufficient upsetting force and overcome the ring resistance to deformation. Besides, it was shown in [2] that for conditions of welding of rings with a significant elasticity it is necessary to take into account the possibility of rupture of welded joint due to elasticity of the ring itself, if upsetting pressure is too quickly decreased after current switching off.

Earlier, it was considered during determination of forging time of welded joint [2] that the value of force, striving to rupture the welded joint, depends only on the forces of elasticity of ring shunting part, tending to impart the ring shape, which it possessed in cold state before joining of edges being welded. However, here the stresses and displacements, predetermined by non-uniformity of temperature field, which arises during performing of welding process as a result of shunting of electric current, were not consid- ered. Temperatures of this field reach their maximum value at the final stage of welding process and then during cooling they begin to decrease slowly. Therefore, the given work is devoted to investigation of influence of temperature field, which is realized in welded ring at the FBW final stage, on the value of upsetting force and force, striving to rupture the joint.

The experimental measurements (Figure 1) showed that in the process of heating and cooling of FB-welded ring, the temperature field arises, which is the function of both radical $r$ as well as circumferential $\theta$ coordinate.

The non-uniformity of distribution of temperature in the circumferential direction in FBW of rings is also visually observed (Figure 2). It is clearly seen that the area of ring being welded, mostly far from clamping devices of welding machine, has a brighter glowing than the areas of shunting part, close to them. This is explained by the fact that clamping devices provide a high heat removal.

The change in temperatures in the controlled spots at the final welding stage is negligible ( $\mathrm{Fi}-$ gure $1, b$ ) therefore, without introducing a great error, it can be considered that the temperature does not depend on time, i.e. the quasi-stationary heat flow is realized [3].

Let us represent the temperature in the form of the Fourier series: 

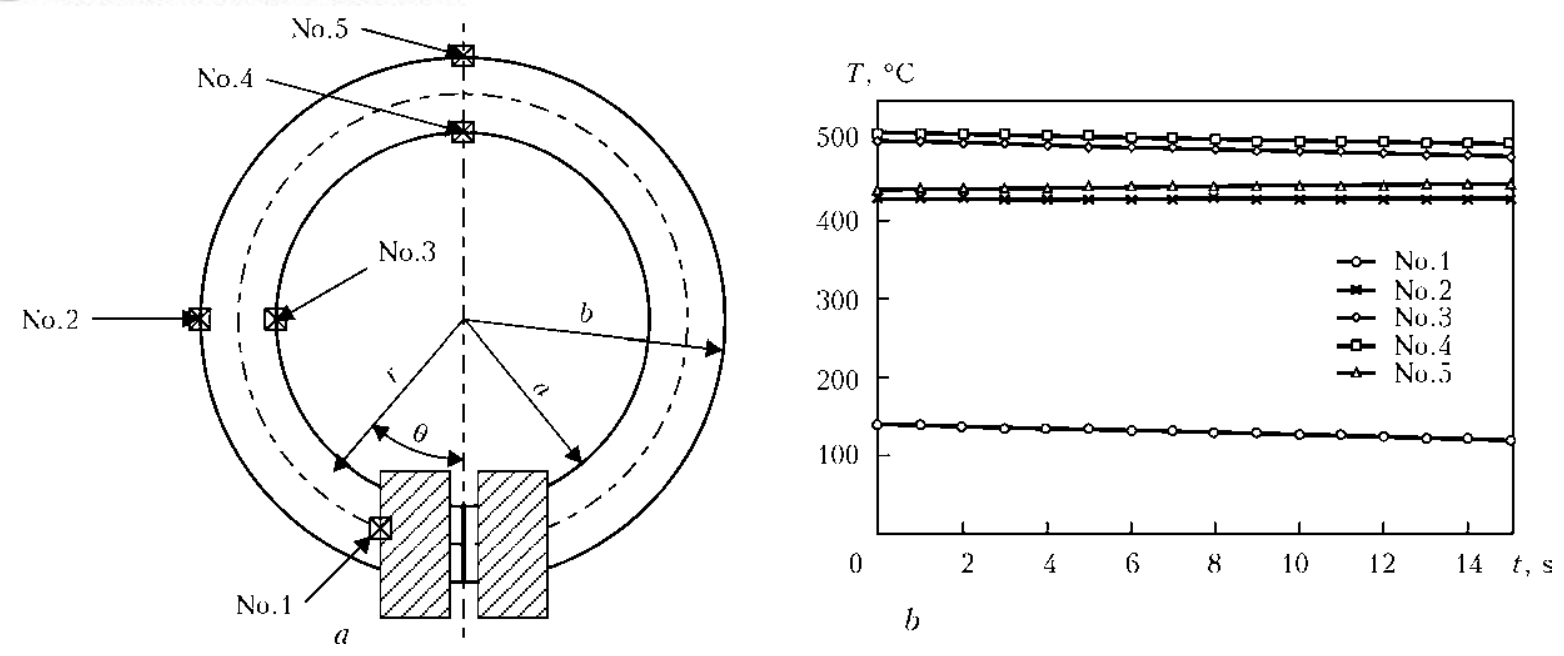

Figure 1. Diagram of location of spots of temperature control at the final stage of welding the ring $(a)$, and dependence of temperatures of controlled spots on time $(b)$

$$
T(r, \theta)=A_{0}(r)+\sum_{1}^{n} A_{n}(r) \cos n \theta,
$$

terms, proportional to $\sin \theta$, are omitted, because the temperature in our case is distributed symmetrically relative to the butt.

To describe the real distribution of temperatures, let us be restricted by the four first terms, included into series (1), which will take the form

$$
\begin{aligned}
& T(r, \theta)=A_{0}(r)+A_{1}(r) \cos \theta+ \\
& +A_{2}(r) \cos 2 \theta+A_{3}(r) \cos 3 \theta .
\end{aligned}
$$

This series corresponds to the experimentally set values in the control spots (see Figure 1) if it meets two systems of equations at $r=a$ and $r=b$ :

$$
\begin{aligned}
& \left\{\begin{array}{l}
A_{0}(a)+A_{1}(a)+A_{2}(a)+A_{3}(a)=T_{1}, \\
A_{0}(a)-A_{2}(a)=T_{3}, \\
A_{0}(a)-A_{1}(a)+A_{2}(a)-A_{3}(a)=T_{4} ;
\end{array}\right. \\
& \left\{\begin{array}{l}
A_{0}(b)+A_{1}(b)+A_{2}(b)+A_{3}(b)=T_{1}, \\
A_{0}(b)-A_{2}(b)=T_{2}, \\
A_{0}(b)-A_{1}(b)+A_{2}(b)-A_{3}(b)=T_{5},
\end{array}\right.
\end{aligned}
$$

where $T_{1}-T_{5}$ are the experimental values of temperatures in the spots Nos $1-5$, respectively (see Figure 1).

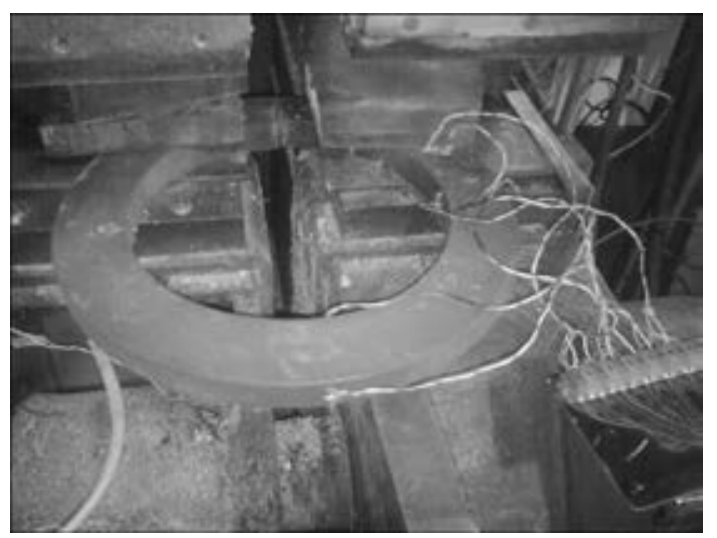

Figure 2. FBW of steel ring with $a=135 \mathrm{~mm}$ and $b=$ $=190 \mathrm{~mm}$ in machine K724

Solution of systems (3) has the following form:

$$
\begin{gathered}
A_{0}(a)=\frac{T_{1}+T_{4}}{4}+\frac{T_{3}}{2}, \\
A_{1}(a)+A_{3}(a)=\frac{T_{1}-T_{4}}{2}, \\
A_{2}(a)=\frac{T_{1}+T_{4}}{4}-\frac{T_{3}}{2}, \quad A_{0}(b)=\frac{T_{1}+T_{5}}{4}+\frac{T_{2}}{2}, \\
A_{1}(b)+A_{3}(b)=\frac{T_{1}-T_{5}}{2}, \quad A_{2}(b)=\frac{T_{1}+T_{5}}{4}-\frac{T_{2}}{2} .
\end{gathered}
$$

It is not difficult to notice that a number of equations in systems (3) is not sufficient to determine the values of all the variables. Therefore. one of variables $A_{1}$ or $A_{3}$ is free. These variables should be selected so that to provide a monotonous growth of temperature in the interval of $\theta$ varying from 0 to $\pi$ and monotonous decrease in the interval of $\theta$ varying from $\pi$ to $2 \pi$.

Substituting the experimental values of temperatures $T_{1}=130, T_{2}=430, T_{3}=480, T_{4}=510$ and $T_{5}=450{ }^{\circ} \mathrm{C}$ to system (3) and selecting the variable $A_{3}$ by corresponding way, we shall obtain the following dependencies describing the changes of temperature in the circumferential direction at external and inner contour:

$$
\begin{aligned}
& T(a, \theta)=400-171 \cos \theta-80 \cos 2 \theta-19 \cos 3 \theta, \\
& T(b, \theta)=360-143 \cos \theta-70 \cos 2 \theta-17 \cos 3 \theta,
\end{aligned}
$$

which are schematically presented in Figure 3.

The change of temperature in the radial direction was determined from the conditions of stationarity of the heat flow [4]

$$
\nabla_{1}^{2} T(r, \theta)=\frac{\partial^{2} T}{\partial r^{2}}+\frac{1}{r} \frac{\partial T}{\partial r}+\frac{1}{r^{2}} \frac{\partial^{2} T}{\partial \theta^{2}}=0 .
$$

Condition (5) can be fulfilled providing that each of the items on the right side (2) meets the following condition:

$$
\begin{gathered}
\nabla_{1}^{2}\left[A_{0}(r)\right]=0 ; \quad \nabla_{1}^{2}\left[A_{1}(r) \cos \theta\right]=0 ; \\
\nabla_{1}^{2}\left[A_{2}(r) \cos 2 \theta\right]=0 ; \quad \nabla_{1}^{2}\left[A_{3}(r) \cos 3 \theta\right]=0 .
\end{gathered}
$$



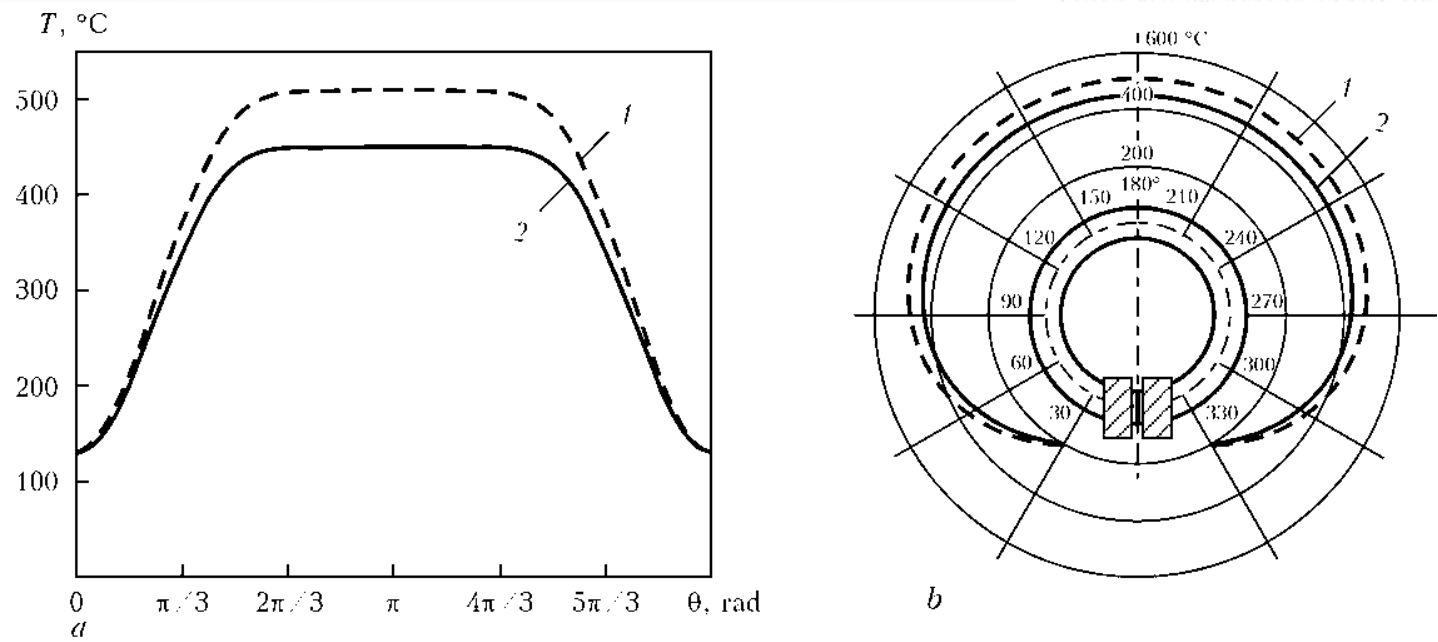

Figure 3. Diagrams of dependence of temperature on circumferential coordinate at $r=a(1)$ and $r=b$ (2) in Cartesian (a) and polar (b) coordinates

Let us represent the solution of equations (6) as follows:

$$
\begin{aligned}
& A_{0}(r)=K_{0}+H_{0} \ln r, \quad A_{1}(r)=K_{1} r+\frac{H_{1}}{r}, \\
& A_{2}(r)=K_{2} r^{2}+\frac{H_{2}}{r^{2}}, \quad A_{3}(r)=K_{3} r^{3}+\frac{H_{3}}{r^{3}},
\end{aligned}
$$

where $K_{i}, H_{i}$ are the constants of integration determined from the conditions $A_{0}(r)=A_{0}(a)$, $A_{1}(r)=A_{1}(a), A_{2}(r)=A_{2}(a), A_{3}(r)=A_{3}(a)$ at $r=a$ and $A_{0}(r)=A_{0}(b), A_{1}(r)=A_{1}(b), A_{2}(r)=$ $=A_{2}(b), A_{3}(r)=A_{3}(b)$ at $r=b$ :

$$
\begin{gathered}
K_{0}=\frac{A_{0}(a) \ln b-A_{0}(b) \ln a}{\ln b-\ln a} ; \\
K_{1}=\frac{1}{b^{2}-a^{2}}\left(b A_{1}(b)-a A_{1}(a)\right) ; \\
K_{2}=\frac{1}{b^{4}-a^{4}}\left(b^{2} A_{2}(b)-a^{2} A_{2}(a)\right) ; \\
K_{3}=\frac{1}{b^{6}-a^{6}}\left(b^{3} A_{3}(b)-a^{3} A_{2}(a)\right) ; \\
H_{0}=\frac{A_{0}(b)-A_{0}(a)}{\ln b-\ln a} ; \\
H_{1}=\frac{a b}{b^{2}-a^{2}}\left(b A_{1}(a)-a A_{1}(b)\right) ; \\
H_{2}=\frac{a^{2} b^{2}}{b^{4}-a^{4}}\left(b^{2} A_{2}(a)-a^{2} A_{2}(b)\right) ; \\
H_{3}=\frac{a^{3} b^{3}}{b^{6}-a^{6}}\left(b^{3} A_{3}(a)-a^{3} A_{3}(b)\right) .
\end{gathered}
$$

Therefore, function describing the change of temperatures in the ring, $T(r, \theta)$, was determined. For experimental data corresponding to the change of temperature in the circumferential direction (see Figure 3), the diagrams of variation of temperatures in the radial direction for different $\theta$ values were plotted (Figure 4).

Determination of temperature stresses acting in the flat ring at the FBW final stage is reduced to the solution of two-dimensional problem of thermoelasticity with stationary heat flow. To solve this problem the similarity between quasistatic problem of thermoelasticity and problem of isothermal theory of elasticity with corresponding volumetric and surface forces, considered in [5], was used.

As it was proved in [6] that the terms of series (1) at $n>1$ have no influence on stressed state, the components of stresses have the following form:

$$
\begin{aligned}
\sigma_{r}= & B_{0} \frac{1}{r^{2}}+2 C_{0}+D_{0}(1+2 \ln r)+ \\
& +\left(2 B_{1} r-\frac{2 C_{1}}{r^{3}}+\frac{D_{1}}{r}\right) \cos \theta, \\
\sigma_{\theta}= & -B_{0} \frac{1}{r^{2}}+2 C_{0}+D_{0}(3+2 \ln r)+ \\
& +\left(6 B_{1} r+\frac{2 C_{1}}{r^{3}}+\frac{D_{1}}{r}\right) \cos \theta, \\
\tau_{r \theta}= & \left(2 B_{1} r-\frac{2 C_{1}}{r^{3}}+\frac{D_{1}}{r}\right) \sin \theta,
\end{aligned}
$$

where $B_{0}, C_{0}, D_{0}$ and $B_{1}, C_{1}, D_{1}$ are the constants of integration.

In our case

$$
\begin{gathered}
B_{0}=-\frac{\alpha E H_{0}}{2\left(b^{2}-a^{2}\right)} a^{2} b^{2} \ln \frac{b}{a}, \quad B_{1}=\frac{\alpha E H_{1}}{4\left(a^{2}+b^{2}\right)}, \\
C_{0}=\frac{\alpha E H_{0}}{4} \frac{b^{2} \ln b-a^{2} \ln a}{b^{2}-a^{2}}+\frac{\alpha E H_{0}}{8}, \\
C_{1}=-\frac{\alpha E H_{1}}{4\left(b^{2}+a^{2}\right)} a^{2} b^{2}, \quad D_{0}=-\frac{\alpha E H_{0}}{4} D_{1}=-\frac{\alpha E H_{1}}{2},
\end{gathered}
$$

where $\alpha$ is the coefficient of linear expansion, $1 /{ }^{\circ} \mathrm{C}$.

It was established in [7] that upsetting force and force, striving to rupture the joint after welding, is related to the value of gap $\delta_{y}$, which represents the sum of primary gap between the edges being welded and shortenings of each of the ends 

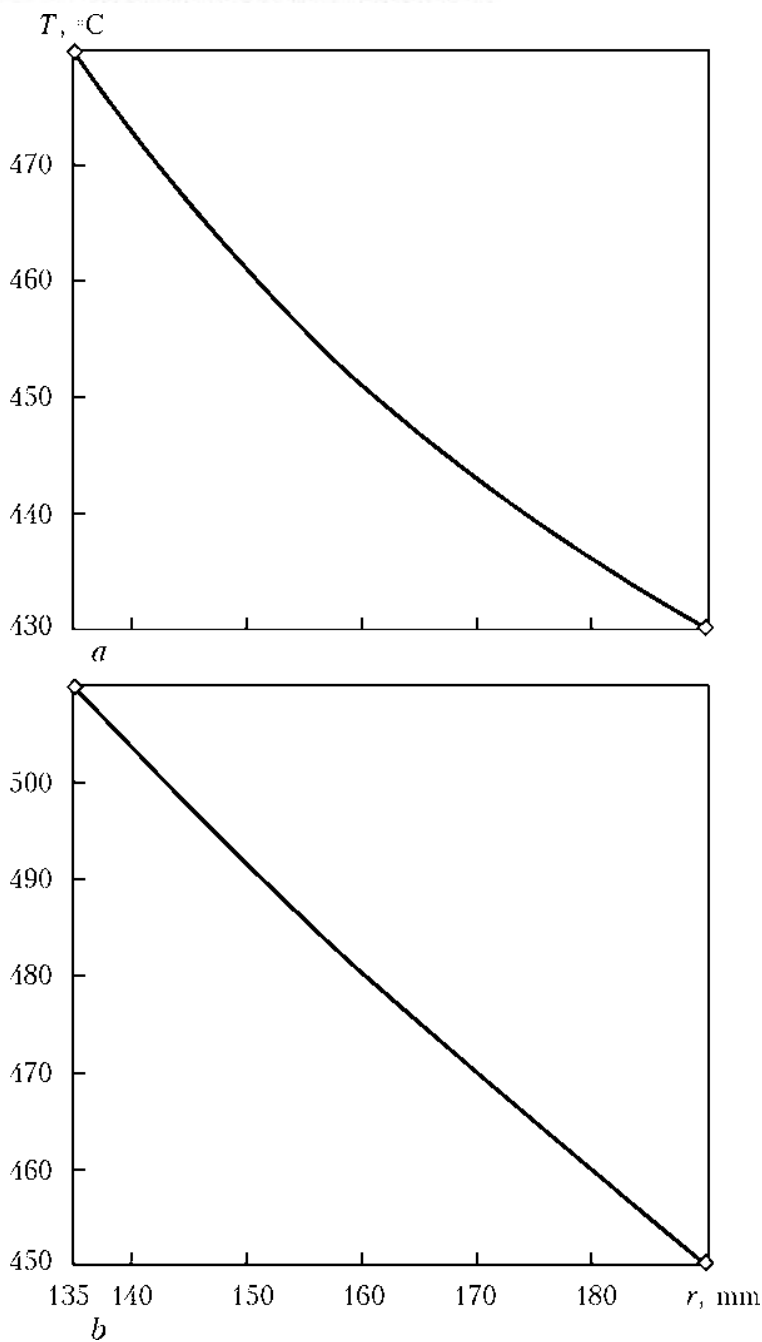

Figure 4. Dependence of temperature on radial coordinate for $\theta=\pi / 2(a)$ and $\theta=\pi(b)$

as a result of flashing and upsetting. However, as the ring is heated, it is necessary to add also displacements of edges being welded, caused by non-uniformity of heating, to the gap values.

As is shown in [6], presence of stresses (9) in the solid ring is equivalent to displacement of edges of ring with gap in the circumferential direction:

$$
\delta_{t}=-2 \pi \alpha\left(H_{0} \frac{a+b}{2}+H_{1}\right) .
$$

Moreover, if $\delta_{t}$ is positive then the gap is increased, and if it is negative then the gap is decreased.

If to calculate the values $-H_{0}$ and $H_{1}$, which correspond to experimental data according to (8), then $\delta_{t}$ is positive, i.e. the gap is increased. This result corresponds to data of investigations carried out in work [6] where it was proved that if in the pipe with cut the temperature of inner surface is higher than that of external one, then distance between the banks of cut is increased.
Upsetting force was calculated according to formulae from [7], and forging time was calculated from data of work [2] with account for displacements of edges being welded, caused by non-uniformity of heating, and also without its account.

For investigated steel ring (steel 20, $\alpha=$ $\left.=1.5 \cdot 10^{-5} 1 /{ }^{\circ} \mathrm{C}, E=2 \cdot 10^{5} \mathrm{MPa}\right)$ the displacement, caused by non-uniformity of heating, amounted to $3.57 \mathrm{~mm}$, that resulted in increase both of necessary upsetting force by $16.5 \%$ and time of forging by $4 \mathrm{~s}$.

\section{Conclusions}

1. It is shown that the Fourier series can be efficiently used to describe the plane temperature fields. In particular, for temperature field, which is realized during cooling of the $\mathrm{FB}$-welded ring, the sufficiently precise description of this field is provided when restricting by four first terms of this series.

2. Analytical dependence was established describing the temperature field in flat ring, which is realized at the final FBW stage. For the steel ring with inner radius $a=135 \mathrm{~mm}$ and external radius $b=190 \mathrm{~mm}$ the diagrams of distribution of temperatures in radial and circumferential directions were plotted.

3. It was grounded and proved by calculations that a part of upsetting force, necessary for formation of joint, is consumed to provide overcoming of elasticity forces caused by non-uniform heating. Therefore, elasticity forces for the case considered, caused by non-uniform heating, result in necessity of increase in upsetting force by $16.5 \%$. Presence of temperature stresses during cooling of ring results also in negligible increase in the force acting in welded joint, due to which forging time is needed to be prolonged by $4 \mathrm{~s}$.

1. Kuchuk-Yatsenko, S.I., Chvertko, P.N., Semyonov, L.A. et al. (2013) Flash butt welding of products of high-strength alloys based on aluminium. The Paton Welding J., 7, 2-6.

2. Kochergin, K.A. (1952) Selection of technology of resistance welding. Leningrad: Sudpromgiz.

3. Carslow, Ch.S., Eger, D. (1964) Heat conductivity of solids. Moscow: Nauka.

4. Boley, B., Weiner, J. (1964) Theory of thermal stresses. Ed. by E.I. Grigolyuk. Moscow: Mir.

5. Timoshenko, S.P. (1937) Theory of elasticity. Leningrad; Moscow: ONTI.

6. Timoshenko, S.P., Goodier, J.N. (1975) Theory of elasticity. Moscow: Mir.

7. Chvertko, P.N., Moltasov, A.V., Samotryasov, S.M. (2014) Calculation of upsetting force in flash butt welding of closed-shape products. The Paton Welding J., 1, 46-50. 